\title{
Günter Germann, Levin Scott, Randolph Sherman: Reconstructive surgery of the hand and upper extremity
}

\section{Thieme Verlag, New York, Stuttgart, Delhi, Rio de Janeiro, 2017, 328 pp. 209 figs., 280.0 mm, Softcover (GEB), EUR (D) 169,99 EUR (A) 174,80 CHF 195,00, ISBN: 978-1-62623-601-1}

\author{
Alain G. Graftiaux ${ }^{1}$ - Pierre Kehr ${ }^{1}$
}

Received: 8 April 2018 / Accepted: 20 May 2018 / Published online: 4 June 2018

(c) Springer-Verlag France SAS, part of Springer Nature 2018

This book (15 years after its first edition) is addressed more particularly to the surgeons who begin in the surgery reconstructive from the upper limb allowing them to find algorithms simple as well as an atlas of the scraps available.

The first chapter relates to the algorithms adapted at the same time at the various stages of the diagnosis but also to the choices of the various techniques and also at the stages of the treatment (lesion of the flexor tendons for example). Illustrated examples are used as a basis for the algorithms of lesions more complex as, for example, for a wound by firearm.

It follows a large chapter from there on the various scraps running, abundantly illustrated more by drawings and photographs.
The book ends in the protocols of rehabilitation and classifications.

On the whole it is a rich book, sometimes a little bulky, which will be useful in all the emergency services to decide in an adequate way of the action to be taken front, during and after the surgical assumption of responsibility.

\section{Compliance with ethical standards}

Conflict of interest The author declares that he has no competing interests.
Pierre Kehr

Strasbourg, France 